\title{
Defect generation by nitrogen during pulsed sputter deposition of GaN
}

Cite as: J. Appl. Phys. 124, 175701 (2018); https://doi.org/10.1063/1.5037340

Submitted: 23 April 2018 . Accepted: 16 October 2018 . Published Online: 01 November 2018

Frederik Steib, Thilo Remmele, Jan Gülink (D), Sönke Fündling, Alexander Behres, Hergo-Heinrich

Wehmann (D), Martin Albrecht $\mathbb{D}^{\mathbb{D}}$, Martin Straßburg, Hans-Jürgen Lugauer $\mathbb{D}^{\mathrm{D}}$, and Andreas Waag

\section{ARTICLES YOU MAY BE INTERESTED IN}

Wide range doping controllability of p-type GaN films prepared via pulsed sputtering Applied Physics Letters 114, 032102 (2019); https://doi.org/10.1063/1.5079673

Electrical properties of Si-doped GaN prepared using pulsed sputtering Applied Physics Letters 110, 042103 (2017); https://doi.org/10.1063/1.4975056

High hole mobility p-type GaN with low residual hydrogen concentration prepared by pulsed sputtering

APL Materials 4, 086103 (2016); https://doi.org/10.1063/1.4960485

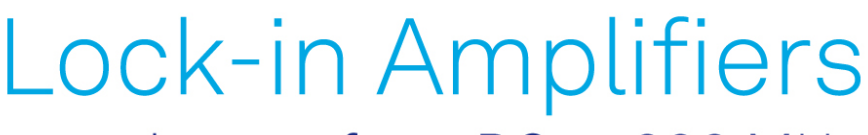

... and more, from DC to $600 \mathrm{MHz}$

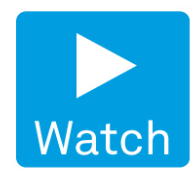




\title{
Defect generation by nitrogen during pulsed sputter deposition of GaN
}

\author{
Frederik Steib, ${ }^{1,2, a)}$ Thilo Remmele, ${ }^{3}$ Jan Gülink, ${ }^{1,2}$ Sönke Fündling, ${ }^{1,2}$ Alexander Behres, ${ }^{4}$ \\ Hergo-Heinrich Wehmann, ${ }^{1,2}$ Martin Albrecht, ${ }^{3}$ Martin Straßburg, ${ }^{4}$ Hans-Jürgen Lugauer, ${ }^{4}$ \\ and Andreas Waag ${ }^{1,2}$ \\ ${ }^{1}$ Institute of Semiconductor Technology and Laboratory for Emerging Nanometrology, Braunschweig \\ University of Technology, 38092 Braunschweig, Germany \\ ${ }^{2}$ Epitaxy Competence Center ec ${ }^{2}$, Hans-Sommer-Straße 66, 38106 Braunschweig, Germany \\ ${ }^{3}$ Leibniz Institute for Crystal Growth, Max-Born-Straße 2, 12489 Berlin, Germany \\ ${ }^{4}$ Osram Opto Semiconductors GmbH, Leibnizstraße 4, 93055 Regensburg, Germany
}

(Received 23 April 2018; accepted 16 October 2018; published online 1 November 2018)

\begin{abstract}
Pulsed sputter deposition has been demonstrated to be a viable process for the growth of high quality $\mathrm{GaN}$ and InGaN/GaN LEDs. It enables the fabrication of nitride LEDs with a red emission wavelength at large areas. In this study, we explore details on the epitaxial sputter deposition of $\mathrm{GaN}$ with a particular emphasis on ion damage. By changing the argon to nitrogen ratio, we adjust the growth mode from island to layer growth. TEM revealed speckles in the epitaxial GaN, which could be identified as isolated basal stacking faults, acting as non-radiative recombination centers. Using Monte Carlo methods, we modeled the energies of backscattered and sputtered atoms in order to get information on the ion damage mechanisms. Considering the collisions on the way from the target to the substrate, we found energetic nitrogen to induce the speckles. A shielding mechanism based on metallic gallium has been identified, leading to the strongly increased luminescence quality in comparison to the non-shielded material. Published by AIP Publishing.

https://doi.org/10.1063/1.5037340
\end{abstract}

\section{INTRODUCTION}

Today, GaN is widely used for LEDs and laser diodes with fundamental emission wavelengths in the ultraviolet and the blue/green part of the optical spectrum. In combination with phosphor converters, they allow for white light emission. As a result, blue GaN LEDs have enabled a revolution in solid state lighting. The standard technique to fabricate group III-nitrides is the metal organic vapour phase epitaxy (MOVPE), due to its relatively high growth rates and good crystal and interface quality of the grown GaN structures. Industrial MOVPE reactors have been scaled up more and more within the past few years to meet the requirements of mass production. Nevertheless, alternative growth techniques with even higher throughput and lower cost structures as well as potentially lower growth temperatures would be desirable. In addition, metal-organic precursors and ammonia also contribute to cost and complexity of MOVPE. Therefore, a growth technique based on metallic gallium $(\mathrm{Ga})$, aluminum, indium, and molecular nitrogen being able to fabricate high quality $\mathrm{GaN}$ at lower temperatures would be very desirable.

Recently, Pulsed Sputter Deposition (PSD) has been promoted to be able to produce InGaN/GaN LEDs on the metal, amorphous materials, and temperature-sensitive substrates. ${ }^{1,2}$ Sputtering fulfills all the advantages mentioned above: lower growth temperatures might be possible, since the atom and molecular species have quite high kinetic energy themselves. Even room temperature growth has been demonstrated. ${ }^{3,4}$ Usually, pure metals and $\mathrm{N}_{2}$ are used in the growth reactor and $\mathrm{GaN}$ layers can be doped with $\mathrm{Ge}$ and $\mathrm{Si}$ for $\mathrm{n}$-doping of

\footnotetext{
${ }^{\text {a) }}$ Author to whom correspondence should be addressed: F.Steib@tu-bs.de
}

the material. Such n-doped films exhibited improved properties with high electron concentrations of above $10^{20} \mathrm{~cm}^{-3}$ and a mobility of above $100 \mathrm{~cm}^{2} \mathrm{~V}^{-1} \mathrm{~s}^{-1}$. 5,6 Furthermore, p-doping was achieved. ${ }^{7}$ These results show the high potential of sputter deposition. In contrast to MOVPE, sputtering is a process that is free of metalorganic precursors and the nitrogen dissociates directly in the plasma, which is used for sputtering the metal target. Therefore, no special nitrogen ion sources are needed. The general scalability of sputtering processes to very large areas and the extremely fast throughput have already been demonstrated in many applications, for example, for the production of silicon solar cells or for the evaporation of reflective coatings on glass windows. ${ }^{8}$ Besides the potential advantages, however, the high kinetic energies of atoms and molecules in the plasma are also one of its major drawbacks, since high energy particles are able to induce defects in the material. This is the reason why sputtering is usually not considered for the fabrication of semiconductor grade materials. In addition, the investigation of defects is a difficult and complex task. Up to now, the issue of defect generation by high-energy particles during PSD of $\mathrm{GaN}$ has only rarely been addressed in the literature.

In this paper, we report on detailed studies on the plasma sputtering of $\mathrm{GaN}$ with a particular focus on defects generated in the GaN semiconductor by high-energy plasma generated species. Panchromatic and spectral cathodoluminescence (CL) investigations are giving insights into the structural and optical quality of the deposited material. The impact of plasma damage has been identified by typical isolated basal stacking faults in high resolution transmission electron microscopy (HRTEM). ${ }^{9}$ In order to understand the basic processes during sputter deposition, we compared our 
experimental results to simulations of ion paths during the gas transport accompanied by additional simulations of the energy transfer in the target, giving information on the spatial and energy distribution of kinetic energies of the sputtered species.

\section{EXPERIMENTAL}

For the sputter growth experiments, a conventional sputter system with a vacuum chamber and a base pressure of about $10^{-6} \mathrm{~Pa}$ has been used. The sputtering target consists of liquid gallium $(7 \mathrm{~N})$ in a molybdenum crucible with 50 mm diameter in a circular IONIX ${ }^{\circledR}$ UHV magnetron sputter source. The setup is in sputtering-up geometry in order to allow liquid gallium to be used as a sputter target. Nitrogen and argon with a purity of $6 \mathrm{~N}$ are used for the deposition with reactive sputtering. The pressure in the chamber has been adjusted by a control valve; in this study pressures of $1 \mathrm{~Pa}$ and $8 \mathrm{~Pa}$ are used. The system is equipped with four different magnetron sources in a co-sputtering geometry for parallel use. A mid-frequency pulsed DC Generator MKS-RPG with a frequency of $250 \mathrm{kHz}$ and a duty cycle of $80 \%$ and MAGPULS QP $1000 / 300$ generator with $100 \mathrm{kHz}$ and a duty cycle of $50 \%$ provide the plasma power. For the work reported here, a sputtering power for Ga of $80 \mathrm{~W}$ is used. During the ON time, the generators apply a negative voltage at the Ga target. Electrons are accelerated and trapped in the magnetic field of the magnetron sources. The electrons ionize neutral gas atoms by collisions. We estimated the ionization ratio of nitrogen by optical emission spectroscopy to be below 5\%. Positive ions are accelerated and sputter the Ga target. The rest of the chamber is connected to the ground. The manipulator with the substrate holder is connected to ground via a $50 \mathrm{Ohm}$ resistor. The substrate is mounted on a molybdenum holder and is heated by a resistive graphite heater encapsulated in boron nitride. Homoepitaxial GaN is sputtered on $5 \mu \mathrm{m}$ GaN buffers fabricated by MOVPE on sapphire. The substrate is thermally cleaned at deposition temperatures in vacuum. Deposition temperatures are set to $730{ }^{\circ} \mathrm{C}$. The distance between target and substrate is fixed at $100 \mathrm{~mm}$ for the experiments in this paper.

The morphology of the layers is investigated with a scanning electron microscopy (SEM) and the optical properties of the semiconductor material by CL measurements at room temperature in a TESCAN MIRA3 GMH system with a GATAN MonoCL4. The structural characterization of the PSD-grown samples was done by HRTEM. The investigations were performed with a FEI Titan 80-300 microscope operated at $300 \mathrm{kV}$ and equipped with an aberration corrected imaging system. Besides classical dark-field and weak-beam techniques for defect characterization, HRTEM lattice imaging under negative spherical aberration conditions ${ }^{9}$ is used to reveal the defect structures in the crystal on an atomic scale. The cross-section samples for HRTEM are prepared by grinding on diamond lapping films and by further thinning of the samples to electron transparency by Ar ion milling under low-incident angles of $4^{\circ}$ with acceleration voltages from $4 \mathrm{kV}$ down to $200 \mathrm{~V}$ to minimize amorphous surface layers.

\section{RESULTS AND DISCUSSION}

Parameters for growth optimization during pulsed plasma sputtering are manifold, and often strongly correlated. For the experiments presented in this paper, we solely have modified the ratio of argon to nitrogen flux, keeping all other growth parameters nominally constant.

The $\mathrm{Ar} / \mathrm{N}_{2}$ directly controls the $\mathrm{Ga} / \mathrm{N}$ ratio during growth. A higher Ar partial pressure leads to an increased $\mathrm{Ga}$ sputter rate on the target. This is caused by the higher atomic mass of $\mathrm{Ar}$ (atomic mass 39.9) in comparison to that of molecular nitrogen (atomic mass 28). The higher mass of $\mathrm{Ar}$ leads to a higher momentum transfer to the heavier $\mathrm{Ga}$ (atomic mass 69.7) atoms, and hence to a higher sputter yield (the relation between energy and momentum transfer and the atomic mass of the participating atoms will be discussed in more detail later). Changing the $\mathrm{Ar} / \mathrm{N}_{2}$ ratio is thus equivalent to changing the III/V ratio, which has a strong influence on the growth behavior.

To get insights into the elementary growth mechanisms during sputtering of $\mathrm{GaN}$, a set of samples has been grown with systematically varying the $\mathrm{Ar} / \mathrm{N}_{2}$ composition in the process gas. SEM micrographs of this set are shown in Fig. 1. For low Ar ratios of $37.5 \%$, a rough surface with pyramidal structures occurs, as seen in Fig. 1(a). By increasing the Ar ratio, the layer morphology becomes smoothened, resulting in larger islands with flat c-facets. However, small voids can be identified at the interface to the GaN buffer indicating that the lateral growth is not perfect [see Fig. 1(b)].

A further increase of the Ar ratio [Fig. 1(c)] to a value of $55 \%$ leads to the formation of liquid gallium droplets on the surface with flat GaN layers in between. Gallium droplet formation is caused by the higher $\mathrm{Ga} / \mathrm{N}$ ratio during growth, see Fig. 1. The Ga droplets do not evaporate during growth since the evaporation rates at temperatures of $730^{\circ} \mathrm{C}$ are too low in comparison to the impingement rate of $\mathrm{Ga}$ atoms. We refer to the conditions of sample $\mathrm{A}$ and $\mathrm{B}$ as being nitrogen-rich, whereas the condition of sample $\mathrm{C}$ is Ga-rich.

These trends are consistent with findings from molecular beam epitaxy (MBE), where low gallium rates lead to threedimensional growth and high Ga rates promote twodimensional layers. ${ }^{10}$ The reason for this behavior is the low surface mobility of the $\mathrm{Ga}$ atoms under N-rich conditions due to the high binding energy of $\mathrm{Ga}-\mathrm{N}$, resulting in pyramidal structures. Under $\mathrm{Ga}$ rich conditions, a Ga ad-layer occurs, leading to a much higher surface mobility of Ga atoms due to a liquid-like behavior. ${ }^{11}$ The adatom mobility during sputter deposition will very likely also depend on the kinetic energy of impinging species, but generally speaking the obtained results are similar to the behavior during MBE growth.

In order to analyze the optical quality of this set of samples, we have performed panchromatic as well as spectral cathodoluminescence (CL) experiments. It turned out that the CL response of samples grown under nitrogen rich conditions is 2 orders of magnitude lower than the optical response from samples grown under Ga-rich conditions, during which Ga droplets are formed.

Samples grown under $\mathrm{Ga}$ rich conditions, forming $\mathrm{Ga}$ droplets, show a pronounced spatial inhomogeneity of their 


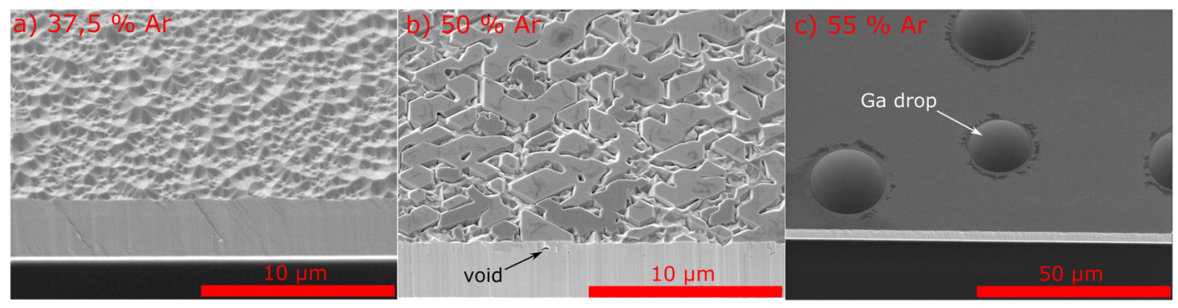

FIG. 1. Plan view SEM micrographs (under an angle of $30^{\circ}$ ) of homoepitaxial sputtered $\mathrm{GaN}$ on MOVPE GaN on sapphire layers grown at different Ar-ratios in the gas phase. At high Ar ratio of $55 \%$, gallium droplets occur on the surface. The thickness of the sputtered $\mathrm{GaN}$ films varies between 750 and $900 \mathrm{~nm}$.

luminescence properties [see Fig. 2(b)]. The regions between the droplets (blue) show a near band edge emission (NBE) at $3.4 \mathrm{eV}$ without blue and yellow band emission, which often occurs in the range of $2.3 \mathrm{eV}$ and $2.8 \mathrm{eV}$, respectively. $\mathrm{CL}$ from regions directly below a $\mathrm{Ga}$ droplet cannot be observed by CL, due to the e-beam and light absorption of the $\mathrm{Ga}$ droplet. However, luckily, regions below the Ga droplets at least partly become visible to CL investigations by Ga droplets moving across the sample surface during growth or by coalescence of Ga droplets after growth, during cool-down. This causes round features (see Fig. 2) in the vicinity of a $\mathrm{Ga}$ droplet, being clearly visible in the CL micrograph. The CL intensity from these regions is much higher than from the flat $\mathrm{GaN}$ regions between $\mathrm{Ga}$ droplets.

The material that was originally positioned under the Ga droplets has been grown under completely different growth conditions, obviously resulting in improved quality, leading to a 10 times brighter NBE as compared to the regions which have not been covered by Ga droplets. The spectrum of GaN grown under a Ga droplet shows in addition to the near band edge emission a blue defect luminescence at around $2.8 \mathrm{eV}$ and yellow luminescence YL at around $2.3 \mathrm{eV}$ (green). It is straightforward to assume that one potential reason for the higher optical efficiency of the GaN layer growing under $\mathrm{Ga}$ droplets is the modified growth mechanism, which would be a vapour liquid solid (VLS) mechanism. In VLS mode, nitrogen diffuses into the Ga droplet forming nitrogen saturated liquid Ga. As a consequence, GaN is formed at the dropletsolid interface. $^{12}$

Similar effects have already been described by Junaid et al. ${ }^{13}$ using high power impulse sputter deposition of GaN on sapphire. The authors observed similar round shapes with improved crystalline quality and identified them as being less strained $\mathrm{GaN}$ by Raman spectroscopy. They found a high number of speckles by TEM in the material outside of the round areas and believed the reason to be energetic bombardment. In the published TEM image are regions visible that are free of speckles and Junaid et al. explained it by point defect diffusion.

The origin of the improved optical quality of $\mathrm{GaN}$ grown under a $\mathrm{Ga}$ droplet is the fact that the $\mathrm{Ga}$ droplet would also shield the underlying GaN from the impact of high energy particles from the plasma process. To identify a potential influence of high energy particles on the defect density, we have performed HRTEM investigations.

The HRTEM measurements of a sample grown under Ga rich conditions are shown in Fig. 3. This sample was grown at a total pressure of $8 \mathrm{~Pa}$ with a high Ar flux of $80 \%$. The PSD GaN was deposited on a standard MOVPE GaN buffer on sapphire substrate. In regions which were not covered by Ga droplets, the interface between the MOVPE GaN buffer and the PSD GaN layer is visible by the onset of speckle contrast in the layer. This speckle pattern can be seen all over the prepared HRTEM sample. The density of the speckles was estimated to be around $10^{17} \mathrm{~cm}^{-3}$ in a thinner area of the sample where the observed volume could be estimated and the number of speckles was reliably quantifiable. We assume that these speckles in the GaN are caused by the impact of high energy particles from the plasma, which will be justified in more detail below.

Analyzing the atomic structure of the speckles from HRTEM images allows identifying them as isolated basal
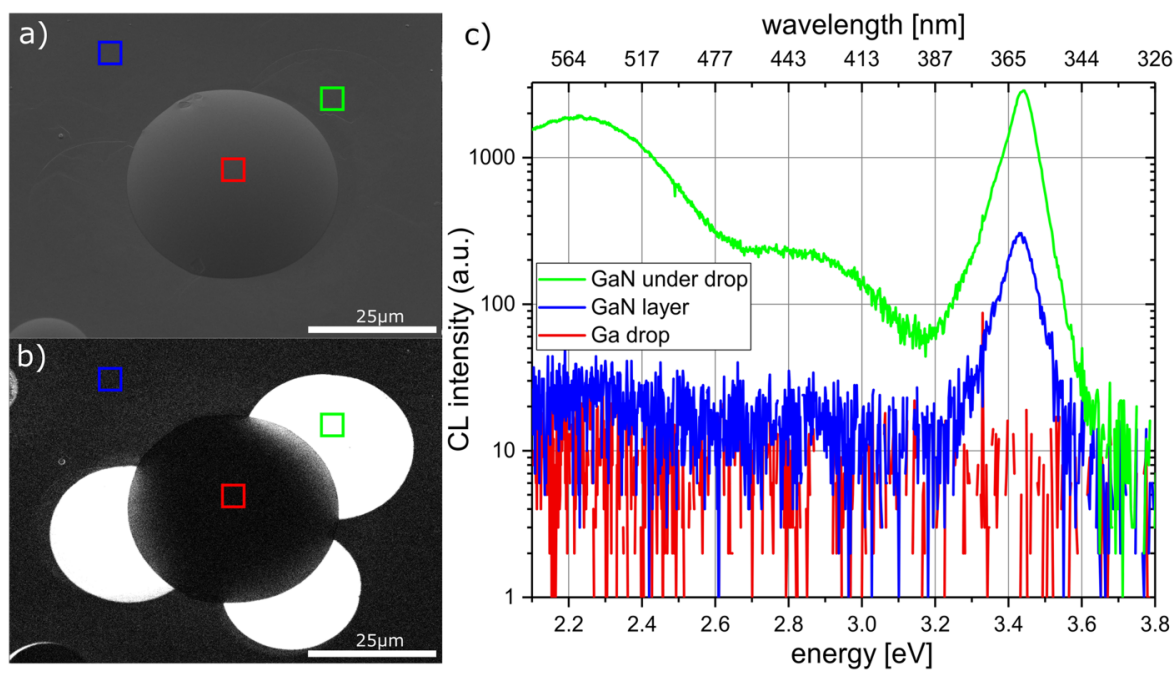

FIG. 2. Secondary electron (a) and panchromatic CL (b) micrograph of a $\mathrm{Ga}$ droplet on the sputtered $\mathrm{GaN}$ on MOVPE GaN with position dependent CL spectra (c) measured at $5 \mathrm{kV}, 250$ $\mathrm{pA}$ and room temperature, showing no $\mathrm{CL}$ luminescence from the gallium droplet (red), a "near band edge emission" (NBE) from the GaN layer (blue) and a spectrum from the $\mathrm{GaN}$ that was protected under a drop (green). The green region shows a 10 times brighter NBE than the blue region and also blue and yellow luminescence. 


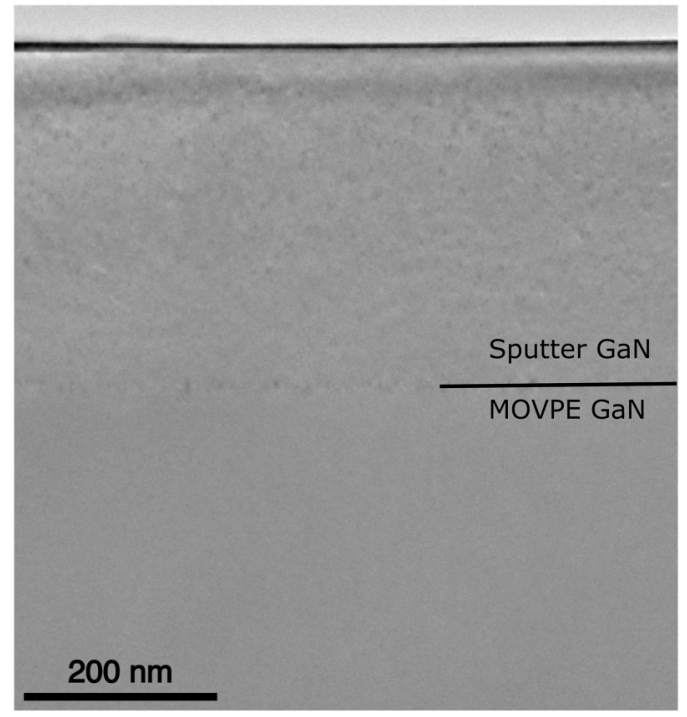

FIG. 3. TEM bright field image along the $<11-20\rangle$ zone axis of a $300 \mathrm{~nm}$ thick, homoepitaxial PSD GaN layer on MOVPE GaN. The marked interface shows a pseudomorphic epitaxial overgrowth of the MOVPE buffer. A speckle pattern with a density of $\sim 10^{17} \mathrm{~cm}^{-3}$ is present in the whole PSD GaN layer.

stacking fault with a lateral extension of around $2 \mathrm{~nm}$ (Fig. 4). This isolated basal stacking fault is an extrinsic stacking fault (type E). These extrinsic stacking faults are known to be formed by precipitation of point defects in the basal plane. ${ }^{14}$ The burgers vector of the confining dislocation loop is estimated by Burgers circuit to be $b=1 / 2\langle 0001\rangle$. These defects can act as non-radiative recombination centres. The isolated stacking faults have already been observed in layers grown by plasma assisted MBE applying an electron cyclotron resonance plasma source by Kirchner et al. ${ }^{15}$ In their paper, the structural properties of $\mathrm{GaN}$ are studied as a function of the ion energy during a plasma MBE growth. These authors observed isolated stacking faults of extrinsic type and of the intrinsic type $I_{1}$ and attribute their formation to high energy nitrogen ions, with a threshold energy for plasma damage of around $100 \mathrm{eV}$. Interestingly, the extent of

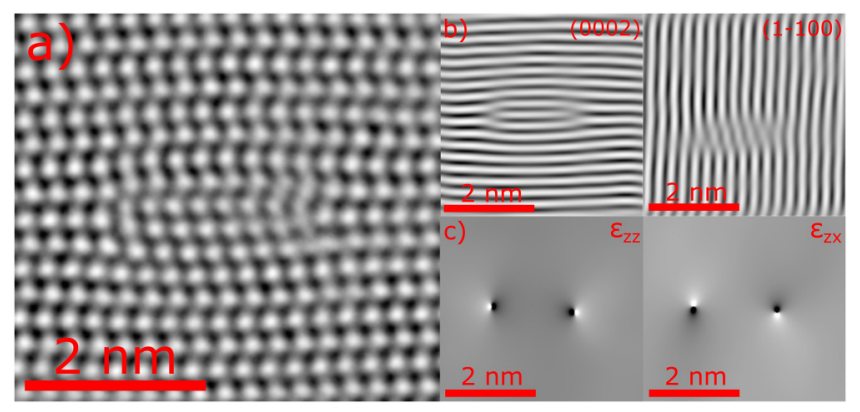

FIG. 4. (a) HRTEM cross sectional micrograph of an isolated basal stacking fault of extrinsic type having a ...ABABCABAB... stacking sequence together with (b) Bragg filtered images showing the (0002) and the (2-200) lattice fringes, respectively. And the (c) strain fields $\varepsilon_{\mathrm{zz}}$ and $\varepsilon_{\mathrm{zx}}$, with $\mathrm{x}=[1-100]$ and $\mathrm{z}=$ [0001] are also shown. While the $\varepsilon_{\mathrm{zz}}$ strain is caused by the dislocation loop with Burgers vector $1 / 2 *[0001]$ confining the extrinsic stacking fault as analyzed by geometrical analysis, the shift of the lattice fringes of the inserted stacking fault layer (the $C$ layer) is visible in the shear strain component $\varepsilon_{\mathrm{zx}}$. the extrinsic faults was around $5 \mathrm{~nm}$ and that of the intrinsic $\mathrm{I}_{1}$ faults around $7 \mathrm{~nm}$, while we observed directly only the extrinsic type with extensions below $3 \mathrm{~nm}$. Based on these experimental findings, we assume that the defects in the PSD grown GaN have the same origin: Impingement of high energy species from the plasma.

An additional hint for the plasma damage deteriorating the optical quality of PSD GaN is the result from Fig. 2, where a much higher optical quality $\mathrm{GaN}$ could be found for $\mathrm{GaN}$ grown below $\mathrm{Ga}$ droplets. Ga droplets will protect the $\mathrm{GaN}$ surface from direct impingement of high-energy plasma species by retarding the neutral atoms and ions. It should be mentioned, however, that the material growing under a $\mathrm{Ga}$ droplet would use a VLS growth mechanism, in contrast to $\mathrm{GaN}$ growing between $\mathrm{Ga}$ droplets. At this stage, the influence of both aspects_-VLS growth and shielding from high energy particles-cannot be discriminated. However, since the penetration depth of high energy particles is quite large, it is safe to assume that ion damage plays an important role in explaining the large differences in optical quality.

In order to get more information on the distribution of high energy ions in the growth reactor, we started simulations to understand the underlying mechanisms more quantitatively. In the MBE experiment of Kirchner et al., the kinetic energy of nitrogen could separately be controlled with a bias voltage $^{15}$ and due to the low pressure, there is no energy relaxation during the transport toward the substrate surface. In the case of PSD, however, the initial ion energies have a broad distribution and both neutral atoms and-due to the much higher pressure-charged species are experiencing a substantial energy relaxation on their way toward the substrate. Therefore, the kinetic energy distributions during PSD are given by a set of external parameters and thus are much less defined and more difficult to control. Parameters that are used to control the energy of the incoming ions are the pressure, the distance between target and substrate, and the sputtering voltage. Since most of the high-energy species are neutral, reducing the energy by retarding electric fields is expected to have only a minor influence.

The transport of ions in matter (TRIM) simulations has been used to estimate the energy of particles during the deposition. TRIM embedded in SRIM 2013 is a Monte Carlo computer program, which calculates interactions between incident ions and target materials. ${ }^{16}$ We simulated a $50 \mathrm{~nm}$ thick gallium target with a normal incidence of ions. The thickness is sufficient that no ions are transmitted through the target. The momentum transfers to the sputtered atoms have been calculated, resulting in kinetic energy and direction of the species. The path of every ion and every energized atom is followed until it has left the target area or until the energy dropped below the smallest binding energy in the target. Various sources of high-energy species during the sputter growth are discussed. On the one hand, ions are backscattered and reflected from the target. On the other hand, Ga atoms are sputtered from the target. Both mechanisms produce high-energy ions and-predominantly-neutral atoms that can potentially induce plasma damage.

On their way to the substrate surface, the particles lose energy due to atom collisions with the plasma gas. 

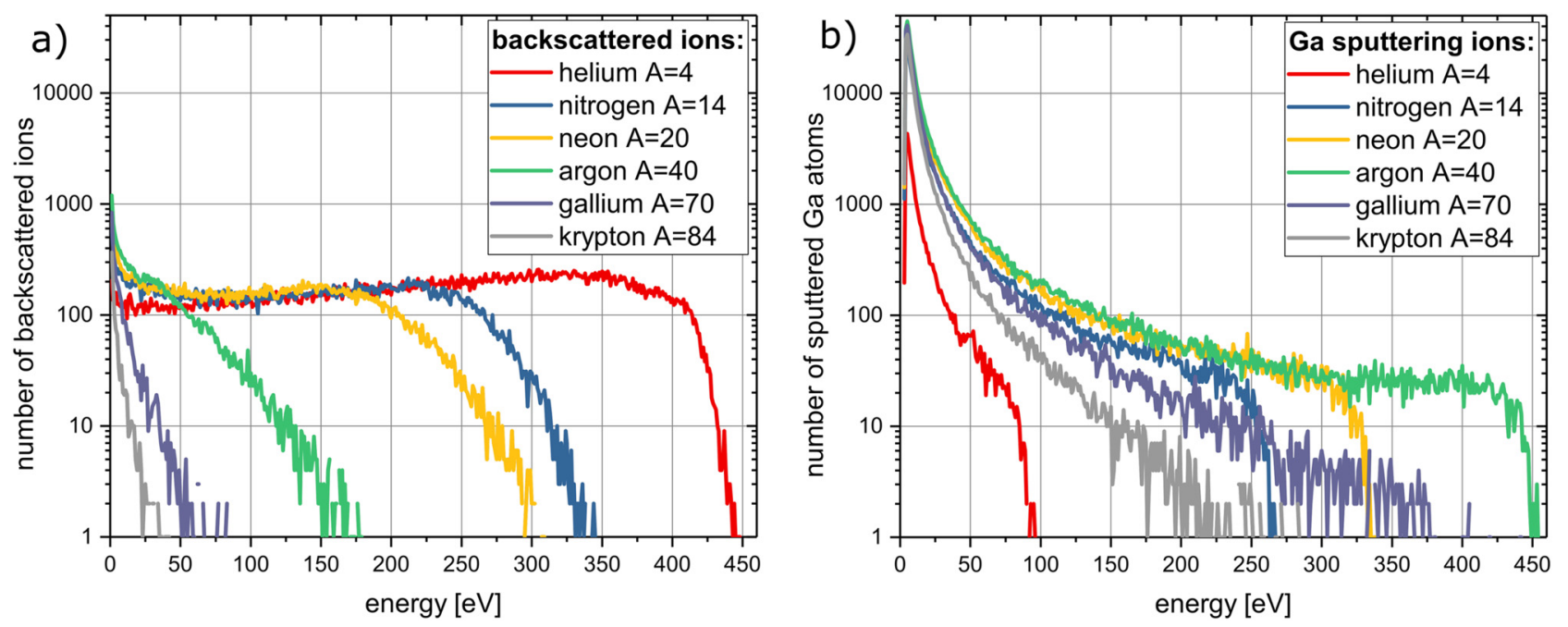

FIG. 5. Energy distribution of backscattered ions with different mass numbers A (a) and sputtered Ga atoms (A = 70); (b) simulated by TRIM, 200000 ions of the listed element with an energy of $500 \mathrm{eV}$ and a normal incidence angle to the gallium target were calculated by the program. The elements are representing the used gas atmosphere during the sputter process. The energy of the atoms is binned with $1 \mathrm{eV}$. The energy of the backscattered ions is higher with lower atomic mass. The energy of the sputtered $\mathrm{Ga}$ atoms is the highest if argon is used.

The retardation depends on the mass of the used gas mixture. As a maximum sputtering voltage we used $500 \mathrm{~V}$, resulting in a maximum energy of $500 \mathrm{eV}$, corresponding to the maximum negative voltage at the target during the process. The simulations are considering the generation of energetic atoms at the target and the transport of high energetic ions through the gas phase. Nitrogen as well as various inert gases like helium, neon, argon, and krypton, which might be suitable for sputtering deposition, were investigated. The species have different atomic mass and hence will modify the energy transfer during atomic collisions. Additionally, gallium was considered, because sputtered gallium can be ionized and then accelerated for self-sputtering.

Figure 5(a) shows the energy spectrum of the ions which are backscattered from the gallium target. As expected, atoms with a small mass are backscattered with higher maximum energy. For monoatomic nitrogen, which is necessary for nitride deposition, the maximum energy is as high as $350 \mathrm{eV}$, which is much higher than the threshold for defect generation of $100 \mathrm{eV}$ as stated above. Figure 5(b) shows the number of ejected $\mathrm{Ga}$ atoms as a function of their kinetic energy when sputtered by various types of sputter ions.

The simulation shows that the majority of sputtered Ga atoms as well as backscattered nitrogen or argon atoms have kinetic energies below the threshold for defect generation of $100 \mathrm{eV} .^{15}$ In Fig. 5, the number of sputtered Ga atoms as well as backscattered atoms with energies above the threshold for defect generation is shown as a function of the type of sputter gas. Table I summarizes the maximum energies for the different sputter gases. Furthermore, the mass number and the sputter yield calculated by the TRIM simulations are given. The energy of backscattered ions is low when they are sputtered with atoms of high atomic mass, see Table I, krypton. The energy of sputtered Ga atoms is low when sputtered with atoms of low atomic mass, see Table I, helium. To judge the potential impact on defect generation, however, the energy relaxation during diffusion to the substrate surface has to be taken into account.

To evaluate the kinetic energies of the incoming species at the substrate surface, the energy relaxation of an atom moving through a sheet of gas at a pressure equal to the operating pressure of the system has been simulated assuming a distance of $100 \mathrm{~mm}$ between target and substrate. In this way, collisions between the sputter and plasma gas atoms in the chamber occur. In this way, the initial kinetic energy of an atom leaving the target area is distributed among many other atoms, and by that the maximum kinetic energy is reduced with each collision. We simulated this transport by TRIM using a gas mixture of $90 \% \mathrm{Ar}$ and $10 \% \mathrm{~N}_{2}$ with a density of $1.7 \times 10^{-4} \mathrm{~kg} \mathrm{~m}^{-3}$ corresponding to a pressure of $10 \mathrm{~Pa}$. Figure 6 shows the energy distribution at the substrate position. For simplicity, an initial kinetic energy of $500 \mathrm{eV}$ was assumed for all ions, even though the results in Fig. 5 show a broad distribution.

Figure 6 shows that practically no gallium atoms with energies above the threshold of $100 \mathrm{eV}$ for generation of isolated basal stacking faults are reaching the substrate after a

TABLE I. Overview of the values for the different elements that were simulated. For every element, the atomic number, the sputter yield, the maximum energy of backscattered ions, and the maximum energy of sputtered Ga atoms are given.

\begin{tabular}{lcccc}
\hline \hline Element & $\begin{array}{c}\text { Mass } \\
\text { number }\end{array}$ & Yield (atoms/ion) & $\begin{array}{c}\text { Maximum } \\
\text { energy of } \\
\text { backscattered } \\
\text { ions (eV) }\end{array}$ & $\begin{array}{c}\text { Maximum } \\
\text { energy of } \\
\text { sputtered Ga } \\
(\mathrm{eV})\end{array}$ \\
\hline $\mathrm{He}$ & 4 & 0.156 & 448 & 98 \\
$\mathrm{~N}$ & 14 & 1.167 & 350 & 268 \\
$\mathrm{Ne}$ & 20 & 1.604 & 310 & 330 \\
$\mathrm{Ar}$ & 40 & 1.890 & 177 & 452 \\
$\mathrm{Kr}$ & 84 & 1.12 & 35 & 283 \\
\hline \hline
\end{tabular}




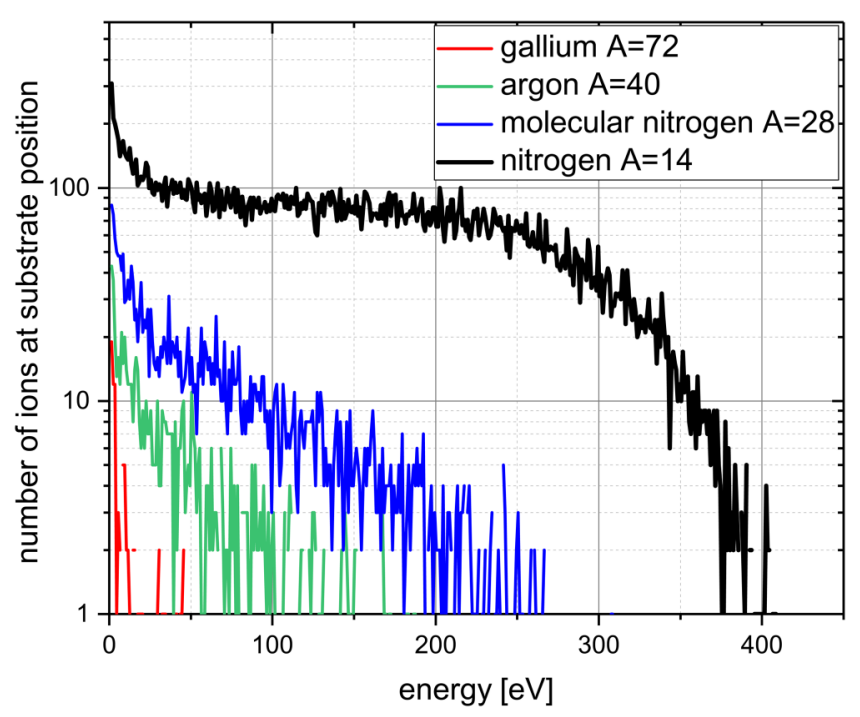

FIG. 6. Energy distribution of monoatomic nitrogen, molecular nitrogen, argon and gallium at a target distance of $100 \mathrm{~mm}$ simulated by TRIM. 200 000 ions of the listed element with an energy of $500 \mathrm{eV}$ were calculated passing $100 \mathrm{~mm}$ of a $90 \% \mathrm{Ar}$ and $10 \% \mathrm{~N}_{2}$ mixture at the pressure of $10 \mathrm{~Pa}$. The energy of the atoms is binned with $1 \mathrm{eV}$.

distance of $100 \mathrm{~mm}$. In contrast to that, nitrogen atoms are less efficiently decelerated during the ballistic transport toward the substrate. In fact, nitrogen is the species which impinges with highest energies. From that, we conclude that nitrogen is the most probable cause for defect generation by high energy particles during nitride plasma deposition.

\section{CONCLUSIONS}

Ion damage has been identified to be a major source for defect formation and hence the low optical material quality during pulsed sputter deposition of GaN. We have identified isolated basal stacking faults in sputtered GaN films as a consequence of sputter damage. From our simulation results of the ballistic transport of high-energy species through the gas phase, we conclude that nitrogen is the main source of high energy particles, and that $\mathrm{Ga}$ atoms or ions play a minor role. Consequently, changing the type of sputter gas will not substantially contribute to improve the structural perfection of the sputtered material. Other strategies for reducing the impact of nitrogen species are increasing the reactor pressure during deposition and/or increase substrate to target distance. Both measures, however, will reduce the growth rates substantially. It depends on the growth reactor and the target area, whether an acceptable compromise between low defect generation and high growth rate can be found.

An alternative strategy is to use surfactants in the form of liquid metal films on the substrate to shield the semiconductor from the impact of high-energy particles. This has been shown by analyzing the optical quality of GaN grown beneath $\mathrm{Ga}$ droplets occurring statistically on the surface during Ga-rich plasma deposition.

When the high quality GaN material can reproducibly be demonstrated by plasma sputtering, it will have a tremendous impact on future GaN technology in general, since this would lead to larger area growth, which in semiconductor technology generally leads to cost reduction. As we have demonstrated, however, key challenges have to be addressed first, including the ion damage caused by high energy atoms during the sputtering process. However, measures have been discussed to reduce this negative impact. A thorough optimisation of the growth reactor and growth process has to be performed in order to fabricate high quality $\mathrm{GaN}$ in a plasma deposition process.

\section{ACKNOWLEDGMENTS}

The authors acknowledge the support of BMBF within the project PlaN B funded by the German Federal Ministry of Education and Research according to the resolution of the German Federal Parliament (BMBF, Support Code 13N13143).

${ }^{1}$ J. W. Shon, J. Ohta, K. Ueno, A. Kobayashi, and H. Fujioka, Sci. Rep. 4, 5325 (2014).

${ }^{2}$ H. Kim, J. Ohta, K. Ueno, A. Kobayashi, and H. Fujioka, Phys. Status Solidi A 214, e201700244 (2017).

${ }^{3}$ K. Sato, J. Ohta, S. Inoue, A. Kobayashi, and H. Fujioka, Appl. Phys. Express 2(1), 011003 (2009).

${ }^{4}$ K. S. Lye, A. Kobayashi, K. Ueno, J. Ohta, and H. Fujioka, Appl. Phys. Lett. 109, 032106 (2016).

${ }^{5}$ K. Ueno, T. Fudetani, Y. Arakawa, A. Kobayashi, J. Ohta, and H. Fujioka, APL Mater. 5, 126102 (2017).

${ }^{6}$ K. Ueno, Y. Arakawa, A. Kobayashi, J. Ohta, and H. Fujioka, Appl. Phys. Express 10(10), 101002 (2017).

${ }^{7}$ Y. Arakawa, K. Ueno, A. Kobayashi, J. Ohta, and H. Fujioka, APL Mater. 4, 086103 (2016).

${ }^{8}$ T. Repmann, S. Wieder, S. Klein, H. Stiebig, and B. Rech, in IEEE 4th World Conference on Photovoltaic Energy Conference (IEEE, Waikoloa, HI, 2006), p. 1724.

${ }^{9}$ C. L. Jia, L. Houben, A. Thust, and J. Barthel, Ultramicroscopy 110(5), 500 (2010).

${ }^{10}$ B. Heying, R. Averbeck, L. F. Chen, E. Haus, H. Riechert, and J. S. Speck, J. Appl. Phys. 88(4), 1855 (2000).

${ }^{11}$ T. Zywietz, J. Neugebauer, and M. Scheffler, Appl. Phys. Lett. 73(4), 487 (1998).

${ }^{12}$ A. Jaud, L. Auvray, A. Kahouli, T. Abi-Tannous, F. Cauwet, G. Ferro, and C. Brylinski, Phys. Status Solidi A 214, 1600428 (2017).

${ }^{13}$ M. Junaid, D. Lundin, J. Palisaitis, C.-L. Hsiao, V. Darakchieva, J. Jensen, POÅ Persson, P. Sandström, W.-J. Lai, L.-C. Chen, K.-H. Chen, U. Helmersson, L. Hultman, and J. Birch, J. Appl. Phys. 110, 123519 (2011).

${ }^{14}$ H. Blank, P. Delavignette, R. Gevers, and S. Amelinckx, Phys. Status Solidi B 7, 747 (1964).

${ }^{15}$ V. Kirchner, H. Heinke, U. Birkle, S. Einfeldt, D. Hommel, H. Selke, and P. L. Ryder, Phys. Rev. B 58, 15749 (1998)

${ }^{16}$ J. F. Ziegler, M. D. Ziegler, and J. P. Biersack, Nucl. Instrum. Methods Phys. Res. B 268(11-12), 1818 (2010). 